\title{
Neutropenia during the First Cycle of Induction Chemotherapy Is Prognostic for Poor Survival in Locoregionally Advanced Nasopharyngeal Carcinoma: A Real-World Study in an Endemic Area
}

\author{
Cheng $\mathrm{Xu}, \mathrm{MD}^{1}$ \\ Shi-Ping Yang, MD² \\ Yuan Zhang, MD' \\ Ling-Long Tang, $M D^{1}$ \\ Guan-Qun Zhou, MD \\ Xu Liu, MD' \\ Yan-Ping Mao, MD' \\ Rui Guo, MD' \\ Wen-Fei Li, MD \\ Lei Chen, MD' \\ Ai-Hua Lin, MD ${ }^{3}$ \\ Ying Sun, MD \\ Jun Ma, MD'
}

${ }^{1}$ Department of Radiation Oncology, Sun Yat-sen University Cancer Center, State Key Laboratory of Oncology in South China, Collaborative Innovation Center for Cancer Medicine, Guangzhou, ${ }^{2}$ Department of Radiology,

Hainan Province People's Hospital, Haikou, ${ }^{3}$ Department of Medical Statistics and Epidemiology, School of Public Health, Sun Yat-sen University, Guangzhou, China

Correspondence: Jun Ma, MD

Department of Radiation Oncology,

Sun Yat-sen University Cancer Center,

State Key Laboratory of Oncology in South

China, Collaborative Innovation Center

of Cancer Medicine, 651 Dongfeng Road East,

Guangzhou 510060, China

Tel: 86-20-87343469

Fax: 86-20-87343295

E-mail: majun2@mail.sysu.edu.cn

Received May 25, 2017

Accepted July 20, 2017

Published Online July 24, 2017

${ }^{*}$ Cheng Xu, Shi-Ping Yang, and Yuan Zhang contributed equally to this work.

\section{Purpose}

The purpose of this study was to investigate the effect of neutropenia during the first cycle of induction chemotherapy (IC-1) on survival in locoregionally advanced nasopharyngeal carcinoma (LANPC).

\section{Materials and Methods}

Eligible patients $(n=545)$ with LANPC receiving IC+concurrent chemoradiotherapy were included. Based on nadir neutrophil after IC-1, all patients were categorized into three groups: no/grade 1-2/grade 3-4 neutropenia. Five-year overall survival (OS) and disease-free survival (DFS) were compared between groups and subgroups stratified by IC regimen. We also explored the occurrence of IC-1-induced myelosuppression events and the minimal value of post-treatment neutrophil-to-lymphocyte ratio (post-NLR $\mathrm{min}_{\text {) }}$. Univariate/multivariate analyses were performed to investigate the effect of IC-1-induced neutropenia, timing of neutropenia, number of myelosuppression events, and high post-NLR min $_{\text {On }}$ OS/DFS.

\section{Results}

Grade 1-2/grade 3-4 neutropenia were associated with poorer OS/DFS than no neutropenia (all $p<0.05$ ); OS/DFS were not significantly different between patients experiencing grade 1-2 vs. 3-4 neutropenia. Neutropenia had no significant effect on OS/DFS in patients receiving docetaxel-cisplatin-5-fluorouracil (TPF). Grade 1-2 (grade 3-4) neutropenia negatively influenced OS/DFS in patients receiving cisplatin-5-fluorouracil (PF) (PF and docetaxel-cisplatin [TP]; all $p<0.05$ ). Neutropenia, two/three myelosuppression events, and high post-NLR $\min (\geq 1.33$ ) was most frequent on days 5-10, second and third week of IC-1, respectively. After adjustment for covariates, IC-1-induced neutropenia, two/three myelosuppression events, and post-NLR $R_{\min } \geq 1.33$ were validated as negative predictors of OS/DFS (all $p<0.05$ ); timing of neutropenia had no significant effect.

\section{Conclusion}

Occurrence of neutropenia, number of myelosuppression events, and high post-NLR min $_{\text {dur- }}$ ing PF/TP IC-1 have prognostic value for poor survival in LANPC.

\author{
Key words \\ Induction chemotherapy, Nasopharyngeal carcinoma, \\ Neutropenia, Lymphocyte, Prognosis, Survival
}




\section{Introduction}

Over $70 \%$ of new cases of nasopharyngeal cancer (NPC) are locoregionally advanced NPC (LANPC; stage III-IV, without distant metastasis) [1]. Concurrent chemoradiotherapy (CCRT) \pm adjuvant chemotherapy is the mainstay treatment. Compared to additional adjuvant chemotherapy, induction chemotherapy (IC) results in early eradication of micrometastases and fewer toxicities; therefore, IC+CCRT is a promising strategy [2].

Myelosuppression (e.g., neutropenia, anemia, thrombocytopenia) is a major chemotherapy side-effect that increases morbidity/mortality and health-care costs [3]. In clinical practice, $30 \%$ of patients experience severe / febrile neutropenia during chemotherapy, two-thirds during the first cycle [4]. The timing of chemotherapy-induced neutropenia (CIN) may affect prognosis [5,6]. Therefore, it is essential to explore the occurrence of CIN and its value in predicting prognosis. However, studies investigating the prognostic value of CIN over multiple cycles on survival in lung/esophageal/colorectal/gynecological cancer have generated positive $[7,8]$, mixed [9], non-significant $[10,11]$ and negative results $[12,13]$. Moreover, CIN after the first cycle has been suggested to indicate chemotherapy resistance, future complications, and poor prognosis [14,15].

CIN may reflect sufficient chemotherapeutic dose, enhanced effectiveness and active tumor response $[9,10,12]$. Alternatively, CIN may reduce host immune function, attenuate resistance to malignant transformation and promote cancer development and progression [12,13,16]. Neutrophilto-lymphocyte ratio (NLR) is a novel joint biomarker, which can comprehensively reflect the level of patients' inflammation and immune function; higher pretreatment NLR has been reported to have association with poorer survival in cancer patients $[17,18]$. However, it still remains unclear whether CIN and post-treatment NLR (post-NLR) can influence survival; no publication has explored this issue in NPC. Moreover, neutropenia during the first cycle of induction chemotherapy (IC-1) merits special attention, as it could indicate high-risk patients who require timely targeted interventions and intensive treatment evaluations.

The primary aim of this study was to investigate the associations between the occurrence and grade of neutropenia during IC-1 and survival in patients with LANPC undergoing IC+CCRT. The secondary aims were to assess the occurrence of myelosuppression events and post-NLR after IC-1 and explore whether the timing of neutropenia, number of myelosuppression events, and high post-NLR could influence survival.

\section{Materials and Methods}

\section{Patient}

Consecutive patients $(n=2,191)$ with newly-diagnosed, non-metastatic, pathologically proven NPC who received radical treatment based on intensity-modulated radiotherapy (IMRT) at Sun Yat-sen University Cancer Center (SYSUCC) between November 2009 and May 2012 in a prospectively maintained database (cutoff time, December 31, 2016) were assessed. As this was a real-world study, we included patients with comorbidities, alcohol consumption, and advanced age [19], via loose eligibility criteria: (1) patients diagnosed with LANPC; (2) patients who underwent IC+CCRT; (3) age of diagnosis $\geq 18$ years-old; (4) no prophylactic use of granulocyte colony-stimulating factor (G-CSF) during IC-1; (5) detailed information on IC-1-induced myelosuppression and other covariates can be collected from the database; 545 patients (24.9\%) were included (S1 Fig.). This study was approved by the Institutional Review Board and Ethics Committee of SYSUCC; the need for informed consent was waived. The authenticity of this article has been validated by uploading the key raw data onto the Research Data Deposit public platform (http:// www.researchdata.org.cn), with the approval RDD number as RDDA2017000145.

\section{Examination, treatment and follow-up}

The following examinations were routinely implemented within the 2-to- 4 weeks prior to IC-1: complete medical history, physical examination, blood and biochemistry tests, nasoendoscopy, neck and nasopharyngeal magnetic resonance imaging, chest radiography, abdominal ultrasonography and whole-body bone scan; ${ }^{18} \mathrm{~F}$-fluorodeoxyglucose positron emission tomography / computed tomography was performed for $187 / 545$ patients $(34.3 \%)$ at initial evaluation. Moreover, the tumor-related biomarkers, i.e., plasma Epstein-Barr virus (EBV) DNA titer, IgA antibodies against viral capsid antigen (VCA-IgA) and early antigen (EA-IgA), were quantified before treatment.

IC was PF ( $80 \mathrm{mg} / \mathrm{m}^{2}$ cisplatin, 4,000 mg/m² 5-fluorouracil), TP (75 mg $/ \mathrm{m}^{2}$ docetaxel, $75 \mathrm{mg} / \mathrm{m}^{2}$ cisplatin), or TPF (60 mg $/ \mathrm{m}^{2}$ docetaxel, $60 \mathrm{mg} / \mathrm{m}^{2}$ cisplatin, 3,000 mg/m² 5 -fluorouracil) every 3 weeks for $2-4$ cycles. The timing of initiation of CCRT is on day 21 after completion of IC. Concurrent chemotherapy was cisplatin $\left(30-40 \mathrm{mg} / \mathrm{m}^{2}\right)$ weekly, or cisplatin (80-100 $\left.\mathrm{mg} / \mathrm{m}^{2}\right)$ every 3 weeks concurrently with IMRT. Full details are provided in the Supplementary Methods. 
Table 1. Baseline characteristics of patients

\begin{tabular}{|c|c|c|c|c|c|}
\hline \multirow[b]{2}{*}{ Characteristic } & \multirow[b]{2}{*}{$\begin{array}{c}\text { No. }(\%)^{a)} \\
(n=545)\end{array}$} & \multicolumn{3}{|c|}{ First-cycle induction chemotherapy-induced neutropenia } & \multirow[b]{2}{*}{ p-value } \\
\hline & & $\begin{array}{c}\left.\text { Absen }^{\mathrm{a}}\right)^{2} \\
(\mathrm{n}=253)\end{array}$ & $\begin{array}{c}\text { Grade 1-2 } \\
(n=182)\end{array}$ & $\begin{array}{c}\text { Grade } 3-4^{a)} \\
(n=110)\end{array}$ & \\
\hline \multicolumn{6}{|c|}{ Age at diagnosis (yr) } \\
\hline $18-36$ & $121(22.2)$ & $60(23.7)$ & $43(23.6)$ & $18(16.4)$ & 0.321 \\
\hline $37-44$ & $138(25.3)$ & $59(23.3)$ & $53(29.1)$ & $26(23.6)$ & \\
\hline $45-51$ & $135(24.8)$ & $63(24.9)$ & $44(24.2)$ & $28(25.5)$ & \\
\hline$\geq 52$ & $151(27.7)$ & $71(28.1)$ & $42(23.1)$ & $38(34.5)$ & \\
\hline \multicolumn{6}{|l|}{ Sex } \\
\hline Male & $418(76.7)$ & $204(80.6)$ & $132(72.5)$ & $82(74.5)$ & 0.120 \\
\hline Female & $127(23.3)$ & $49(19.4)$ & $50(27.5)$ & $28(25.5)$ & \\
\hline \multicolumn{6}{|c|}{ Family history of cancer } \\
\hline No & $412(75.6)$ & $194(76.7)$ & $138(75.8)$ & $80(72.7)$ & 0.720 \\
\hline Yes & $133(24.4)$ & $59(23.3)$ & $44(24.2)$ & $30(27.3)$ & \\
\hline \multicolumn{6}{|l|}{ Comorbidity } \\
\hline No & $382(70.1)$ & $167(66.0)$ & $137(75.3)$ & $78(70.9)$ & 0.112 \\
\hline Yes & $163(29.9)$ & $86(34.0)$ & $45(24.7)$ & $32(29.1)$ & \\
\hline \multicolumn{6}{|c|}{ Cigarette smoking } \\
\hline No & $328(60.2)$ & $150(59.3)$ & $112(61.5)$ & $66(60.0)$ & 0.893 \\
\hline Yes & $217(39.8)$ & $103(40.7)$ & $70(38.5)$ & $44(40.0)$ & \\
\hline \multicolumn{6}{|c|}{ WHO histologic type } \\
\hline Type I-II & $26(4.8)$ & $11(4.3)$ & $8(4.4)$ & $7(6.4)$ & 0.680 \\
\hline Type III & $519(95.2)$ & $242(95.7)$ & $174(95.6)$ & $103(93.6)$ & \\
\hline \multicolumn{6}{|c|}{ Viral capsid antigen $\operatorname{Ig} A^{b)}$} \\
\hline$<1: 80$ & $161(29.5)$ & $72(28.5)$ & $54(29.7)$ & $35(31.8)$ & 0.885 \\
\hline $1: 80-1: 320$ & $266(48.8)$ & $126(49.8)$ & $91(50.0)$ & $49(44.5)$ & \\
\hline$\geq 1: 640$ & $118(21.7)$ & $55(21.7)$ & $37(20.3)$ & $26(23.6)$ & \\
\hline \multicolumn{6}{|c|}{ Early antigen $\operatorname{Ig} A^{b)}$} \\
\hline$<1: 10$ & $205(37.6)$ & 95 (37.5) & $70(38.5)$ & $40(36.4)$ & 0.977 \\
\hline 1:10-1:20 & $143(26.2)$ & $69(27.3)$ & $46(25.3)$ & $28(25.5)$ & \\
\hline$\geq 1: 40$ & $197(36.1)$ & $89(35.2)$ & $66(36.3)$ & $42(38.2)$ & \\
\hline \multicolumn{6}{|c|}{ Epstein-Barr virus DNA titer (copy/mL) ${ }^{\text {b) }}$} \\
\hline$<10,000$ & $302(55.4)$ & $142(56.1)$ & $102(56.0)$ & $58(52.7)$ & 0.981 \\
\hline $10,000-99,999$ & $183(33.6)$ & $84(33.2)$ & $60(33.0)$ & $39(35.5)$ & \\
\hline$\geq 100,000$ & $60(11.0)$ & $27(10.7)$ & $20(11.0)$ & $13(11.8)$ & \\
\hline \multicolumn{6}{|c|}{ Neutrophil-to-lymphocyte ratio ${ }^{\text {b) }}$} \\
\hline$<2.35$ & $272(49.9)$ & $128(50.6)$ & $86(47.3)$ & $58(52.7)$ & 0.634 \\
\hline$\geq 2.35$ & $273(50.1)$ & $125(49.4)$ & $96(52.7)$ & $52(47.3)$ & \\
\hline \multicolumn{6}{|c|}{ Clinical stage (8th edition) } \\
\hline III & $249(45.7)$ & $114(45.1)$ & $81(44.5)$ & $54(49.1)$ & 0.720 \\
\hline IVA & $296(54.3)$ & $139(54.9)$ & $101(55.5)$ & $56(50.9)$ & \\
\hline \multicolumn{6}{|c|}{ T category (8th edition) ${ }^{c}$} \\
\hline $\mathrm{T} 1$ & $20(3.7)$ & $3(1.2)$ & $9(4.9)$ & $8(7.3)$ & 0.113 \\
\hline $\mathrm{T} 2$ & $50(9.2)$ & $25(9.9)$ & $17(9.3)$ & $8(7.3)$ & \\
\hline $\mathrm{T} 3$ & $292(53.6)$ & $137(54.2)$ & $94(51.6)$ & $61(55.5)$ & \\
\hline $\mathrm{T} 4$ & $183(33.6)$ & $88(34.8)$ & $62(34.1)$ & $33(30.0)$ & \\
\hline \multicolumn{6}{|c|}{$\mathrm{N}$ category (8th edition) ${ }^{\mathrm{c}}$} \\
\hline No & $35(6.4)$ & $18(7.1)$ & $10(5.5)$ & $7(6.4)$ & 0.928 \\
\hline N1 & $270(49.5)$ & $120(47.4)$ & $91(50.0)$ & 59 (53.6) & \\
\hline N2 & $101(18.5)$ & $50(19.8)$ & 32 (17.6) & 19 (17.3) & \\
\hline N3 & $139(25.5)$ & $65(25.7)$ & $49(26.9)$ & $25(22.7)$ & \\
\hline
\end{tabular}

(Continued to the next page) 
Table 1. Continued

\begin{tabular}{|c|c|c|c|c|c|}
\hline \multirow[b]{2}{*}{ Characteristic } & \multirow[b]{2}{*}{$\begin{array}{l}\text { No. }(\%)^{a)} \\
(n=545)\end{array}$} & \multicolumn{3}{|c|}{ First-cycle induction chemotherapy-induced neutropenia } & \multirow[b]{2}{*}{ p-value } \\
\hline & & $\begin{array}{c}\text { Absent }^{a)} \\
(n=253)\end{array}$ & $\begin{array}{c}{\text { Grade } 1-2^{a)}}^{(n=182)}\end{array}$ & $\begin{array}{c}\text { Grade } 3-4^{a)} \\
(n=110)\end{array}$ & \\
\hline \multicolumn{6}{|c|}{ Chemotherapy regimen } \\
\hline PF & $121(22.2)$ & $78(30.8)$ & $31(17.0)$ & $12(10.9)$ & $<0.001$ \\
\hline $\mathrm{TPF}$ & $230(42.2)$ & $84(33.2)$ & $88(48.4)$ & $58(52.7)$ & \\
\hline $\mathrm{TP}$ & $194(35.6)$ & $91(36.0)$ & $63(34.6)$ & $40(36.4)$ & \\
\hline
\end{tabular}

Values are presented as number (\%). WHO, World Health Organization; PF, cisplatin-5-fluorouracil; TPF, docetaxel-cisplatin5-fluorouracil; TP, docetaxel-cisplatin. a)Percentages may not add up to 100 because of rounding, b) All variables were measured before treatment, ${ }^{c}$ 8th edition of the Union for International Cancer Control/American Joint Committee on Cancer staging system.

\section{Assessment of neutropenia during IC-1}

All patients underwent weekly blood and biochemistry tests for safety surveillance during the 21-day IC-1 cycle. G-CSF was provided whenever possible for patients suffering grade 3-4/febrile neutropenia; additional blood tests were used to monitor recovery status. Each patient had at least three tests during IC-1.

Myelosuppression events was classified using National Cancer Institute Common Terminology Criteria for Adverse Events (ver. 4.0) based on nadir absolute neutrophil count (ANC), hemoglobin concentration $(\mathrm{Hb})$, and platelet count (PLT). Neutropenia was categorized as no neutropenia (shown as 'absent' in figures/tables; ANC $\geq 2.0 \times 10^{9} / \mathrm{L}$ ), grade $1-2\left(1.0 \times 10^{9} / \mathrm{L} \leq \mathrm{ANC}<2.0 \times 10^{9} / \mathrm{L}\right.$ ) or grade $3-4$ (ANC $<1.0 \times 10^{9} / \mathrm{L}$ ); anemia, nadir $\mathrm{Hb}<115.0 \mathrm{~g} / \mathrm{L}$; and thrombocytopenia, nadir PLT $<100.0 \times 10^{9} / \mathrm{L}$. Timing of neutropenia was classified as: first (days 1-7), second (days 8-14), and third (days 15-21) week of IC-1. Number of myelosuppression events was classified as: group 1 (none), group 2 (one), and group 3 (two/three). As G-CSF can substantially increase ANC and make the maximal and average value of post-NLR deviate from the real level of natural course, we used the minimal value of post-NLR (post-NLR $R_{\min }$ ) to perform analysis. Post-NLR min more than or equal to median was defined as high level.

End-points were overall survival (OS) and disease-free survival (DFS). OS was defined as the duration from the date of treatment to death from any cause or last date known alive. DFS was defined as the duration from the date of treatment to failure (i.e., locoregional relapse and distant metastasis), death or last follow-up, whichever happened first.

\section{Statistical analysis}

Follow-up was reported as median value and range; cate- gorical variables, as frequencies and percentages. Descriptive statistics provided as continuous variables were converted into categorical variables according to interquartile range (IQR; age at diagnosis), median value (pretreatment NLR and post-NLR ${ }_{\min }$ ), and cutoff value (EBV DNA, VCA-IgA, and EA-IgA), as reported previously [17]. Baseline characteristics were compared using the chi-square test; actuarial 5-year OS/DFS, estimated using the Kaplan-Meier method and compared using the log-rank test [20]. Neutropenia, anemia, thrombocytopenia, and post-NLR min $_{\text {were considered }}$ time-to-event data; cumulative incidences were calculated with the Kaplan-Meier method and compared with the logrank test [20], and presented using Microsoft Excel (Microsoft Inc., Redmond, WA).

Significant covariates in univariate analysis were entered into multivariate Cox regression analyses using the backward stepwise algorithm (see Supplementary Methods). Adjusted hazard ratios (AHRs) and 95\% confidence intervals were calculated for multivariate analysis, and presented as forest plots using Microsoft Excel via Neyeloff's method [21]. Statistical analyses and figures were generated using SPSS ver. 22.0 (IBM Corp., Armonk, NY), unless otherwise specified. Two-sided $p$-values of $<0.05$ were considered significant.

\section{Results}

\section{Baseline characteristics}

Median age of the 545 eligible patients was 45 years (IQR, 37 to 52) (Table 1); male-to-female ratio was approximately 3:1; $163 / 545$ patients $(29.9 \%)$ had at least one comorbidity; and 519/545 (95.2\%) had non-keratinizing undifferentiated 
A

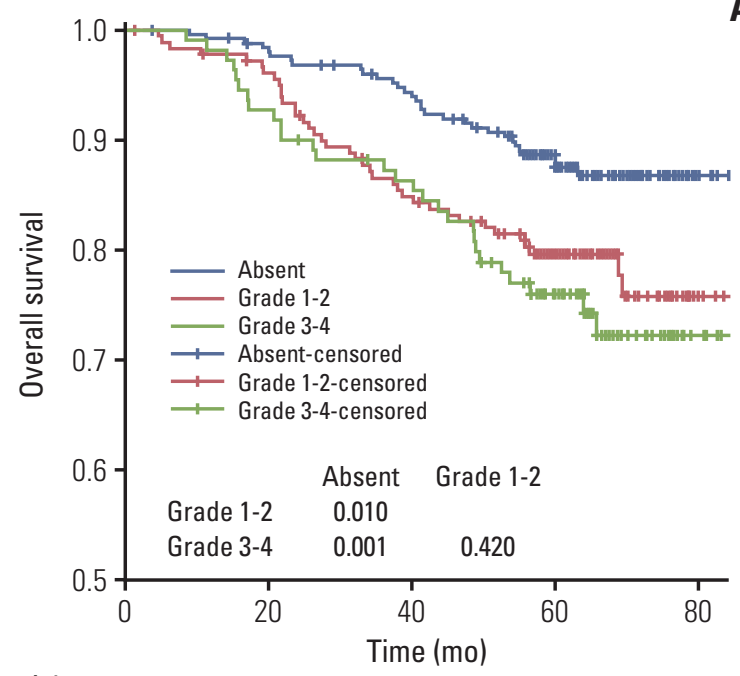

No. at risk

$\begin{array}{rrrrrr}\text { Absent } & 252 & 244 & 201 & 84 & 6 \\ \text { Grade 1-2 } & 181 & 171 & 130 & 53 & 2 \\ \text { Grade 3-4 } & 110 & 101 & 80 & 32 & 3\end{array}$

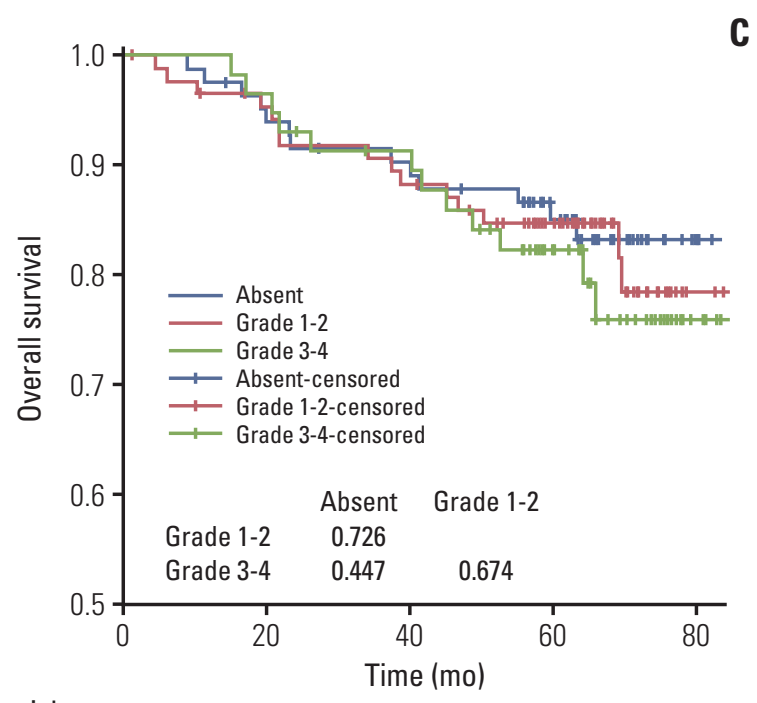

No. at risk

$\begin{array}{rrrrrr}\text { Absent } & 84 & 78 & 66 & 30 & 2 \\ \text { Grade 1-2 } & 87 & 81 & 67 & 30 & 1 \\ \text { Grade 3-4 } & 58 & 55 & 44 & 20 & 3\end{array}$

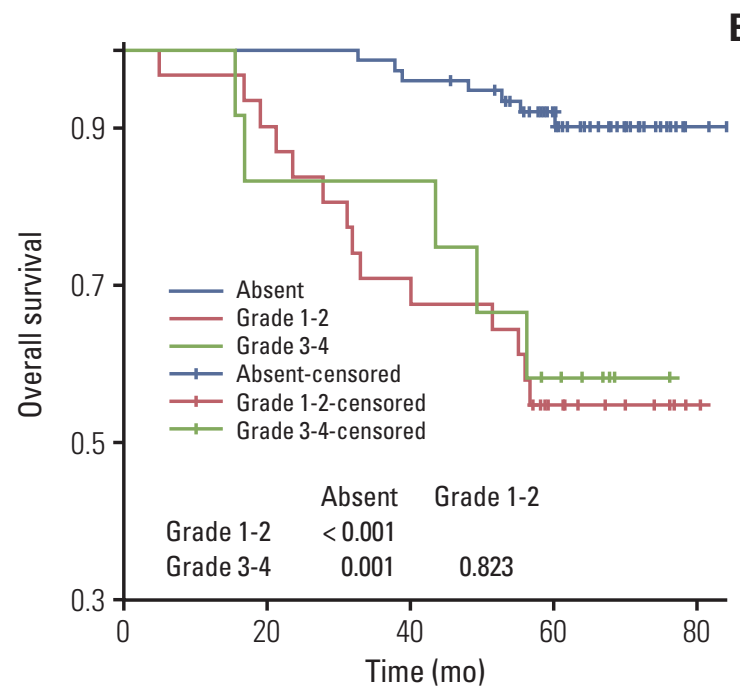

$\begin{array}{rrrrrr}\text { No. at risk } & & & & & \\ \text { Absent } & 78 & 78 & 65 & 29 & 3 \\ \text { Grade 1-2 } & 31 & 28 & 19 & 6 & 5 \\ \text { Grade 3-4 } & 12 & 10 & 10 & 3 & 0\end{array}$

Fig. 1. Kaplan-Meier survival curves for overall survival (A-D) and disease-free survival (E-H) stratified by grade of neutropenia. The columns represent subgroups receiving PF (B, F), TPF (C, G), and TP (D, H), respectively. PF, cisplatin-5-fluorouracil; TPF, docetaxel-cisplatin-5-fluorouracil; TP, docetaxel-cisplatin. (Continued to the next page)

NPC (i.e., World Health Organization [WHO] histologic type III), typical of the endemic area. Most patients received TPF $(42.2 \%)$ or TP regimen $(35.6 \%) ; 22.2 \%$ received PF. All base- line characteristics were well-balanced between patients with no neutropenia, grade 1-2 and grade 3-4 neutropenia, except for chemotherapy regimen $(\mathrm{p}<0.001)$. After median follow- 


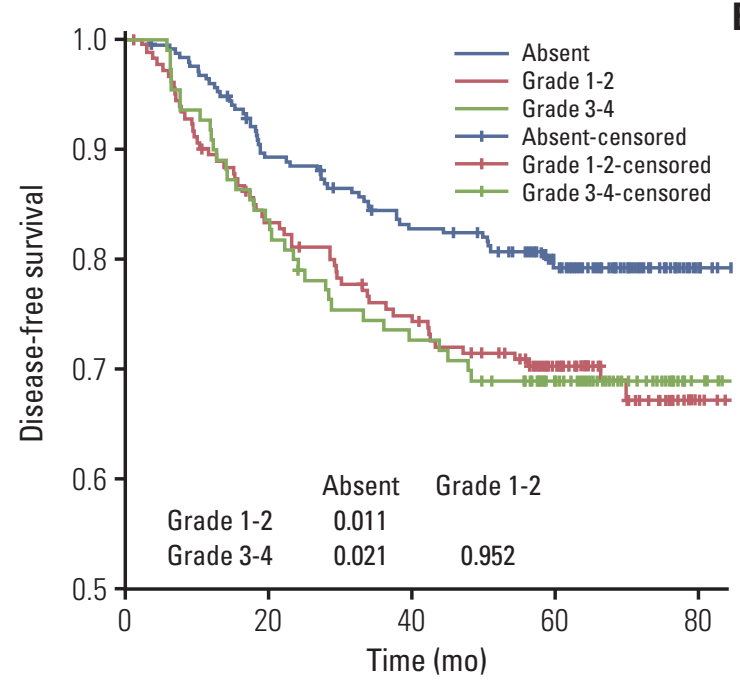

No. at risk

$\begin{array}{rrrrrr}\text { Absent } & 256 & 221 & 175 & 76 & 6 \\ \text { Grade 1-2 } & 181 & 148 & 115 & 48 & 2 \\ \text { Grade 3-4 } & 110 & 92 & 67 & 29 & 3\end{array}$

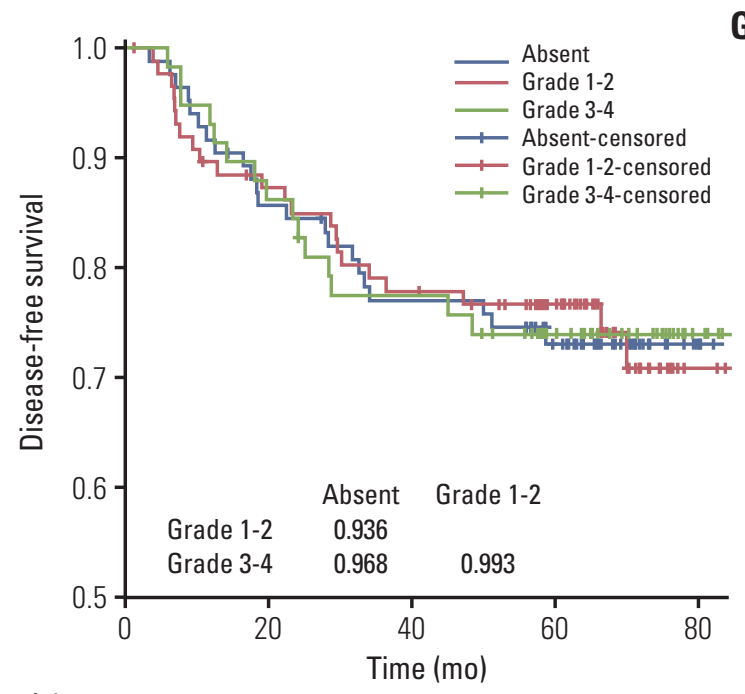

G

No. at risk

$\begin{array}{rlllll}\text { Absent } & 84 & 70 & 57 & 26 & 2 \\ \text { Grade 1-2 } & 87 & 74 & 59 & 27 & 1 \\ \text { Grade 3-4 } & 58 & 50 & 38 & 17 & 3\end{array}$

Fig. 1. (Continued from the previous page)

up of 63 months (range, 1 to 85 months), 97/545 patients $(17.8 \%)$ had died; 60/545 (11.0\%) and 90/545 (16.5\%) developed locoregional relapse and distant metastases, respectively.

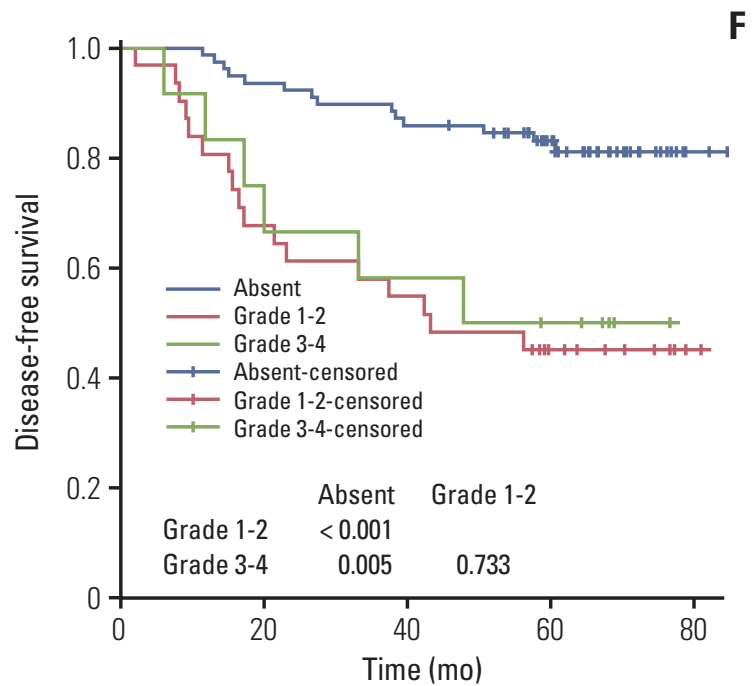

No. at risk

$\begin{array}{rrrrrr}\text { Absent } & 78 & 73 & 58 & 26 & 3 \\ \text { Grade 1-2 } & 31 & 21 & 15 & 6 & 5 \\ \text { Grade 3-4 } & 12 & 9 & 7 & 3 & 0\end{array}$

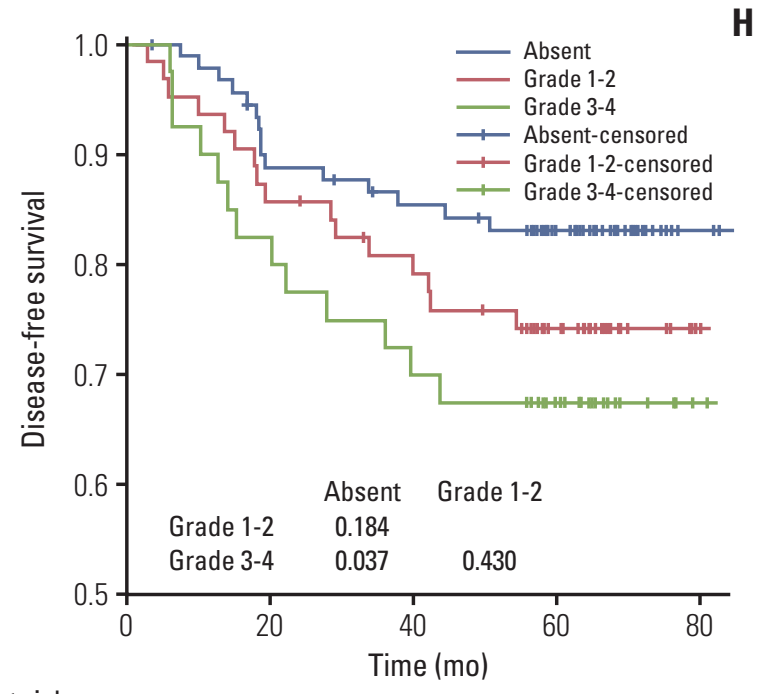

No. at risk

$\begin{array}{rrrrrr}\text { Absent } & 90 & 78 & 61 & 25 & 2 \\ \text { Grade 1-2 } & 63 & 53 & 41 & 15 & 5 \\ \text { Grade 3-4 } & 40 & 33 & 23 & 9 & 5\end{array}$

\section{Association between grade of neutropenia and survival}

Patients without neutropenia had better OS/DFS than patients with grade $1-2(p=0.010$ and $p=0.011)$ and grade $3-4$ $(p=0.001$ and $p=0.021)$ neutropenia (Fig. 1). OS /DFS were not 
Table 2. Univariate and multivariate analysis of the effect of grade of neutropenia on survival

\begin{tabular}{|c|c|c|c|c|c|c|c|c|}
\hline \multirow{3}{*}{ Variable } & \multicolumn{4}{|c|}{ Overall survival } & \multicolumn{4}{|c|}{ Disease-free survival } \\
\hline & \multicolumn{2}{|c|}{ Univariate } & \multicolumn{2}{|c|}{ Multivariate } & \multicolumn{2}{|c|}{ Univariate } & \multicolumn{2}{|c|}{ Multivariate } \\
\hline & HR $(95 \%$ CI) & p-value & AHR (95\% CI) & p-value & HR $(95 \%$ CI $)$ & p-value & AHR $(95 \%$ CI) & p-value \\
\hline \multicolumn{9}{|l|}{ Age at diagnosis (yr) } \\
\hline $18-36$ & Reference & & - & & Reference & & - & \\
\hline $37-44$ & $1.02(0.56-1.85)$ & 0.952 & - & - & $1.19(0.72-1.96)$ & 0.500 & - & - \\
\hline $45-51$ & $1.07(0.59-1.94)$ & 0.837 & - & - & $1.21(0.73-1.99)$ & 0.459 & - & - \\
\hline$\geq 52$ & $1.37(0.78-2.40)$ & 0.272 & - & - & $1.26(0.78-2.06)$ & 0.345 & - & - \\
\hline \multicolumn{9}{|l|}{ Sex } \\
\hline Female vs. male (ref.) & $0.94(0.59-1.52)$ & 0.808 & - & - & $1.06(0.72-1.56)$ & 0.774 & - & - \\
\hline \multicolumn{9}{|l|}{ Family history of cancer } \\
\hline Yes vs. no (ref.) & $1.01(0.64-1.60)$ & 0.961 & - & - & $0.95(0.64-1.40)$ & 0.781 & - & - \\
\hline \multicolumn{9}{|l|}{ Comorbidity } \\
\hline Yes vs. no (ref.) & $0.84(0.53-1.32)$ & 0.454 & - & - & $0.76(0.52-1.11)$ & 0.155 & - & - \\
\hline \multicolumn{9}{|l|}{ Cigarette smoking } \\
\hline Yes vs. no (ref.) & $1.19(0.80-1.78)$ & 0.396 & - & - & $1.06(0.76-1.49)$ & 0.721 & - & - \\
\hline \multicolumn{9}{|l|}{ WHO histologic type } \\
\hline III vs. I-II (ref.) & $0.42(0.21-0.84)$ & 0.014 & $0.47(0.23-0.95)$ & 0.034 & $0.48(0.27-0.86)$ & 0.015 & $0.50(0.28-0.92)$ & 0.025 \\
\hline \multicolumn{9}{|l|}{ Viral capsid antigen $\operatorname{Ig} A^{a)}$} \\
\hline$<1: 80$ & Reference & & - & & Reference & & - & \\
\hline $1: 80-1: 320$ & $0.88(0.56-1.40)$ & 0.600 & - & - & $0.88(0.59-1.29)$ & 0.499 & - & - \\
\hline$\geq 1: 640$ & $0.83(0.47-1.47)$ & 0.524 & - & - & $0.97(0.61-1.54)$ & 0.910 & - & - \\
\hline \multicolumn{9}{|l|}{ Early antigen $\operatorname{Ig} A^{a)}$} \\
\hline$<1: 10$ & Reference & & - & & Reference & & - & \\
\hline 1:10-1:20 & $0.96(0.58-1.58)$ & 0.864 & - & - & $0.79(0.51-1.21)$ & 0.279 & - & - \\
\hline$\geq 1: 40$ & $0.89(0.56-1.43)$ & 0.638 & - & - & $0.88(0.60-1.29)$ & 0.520 & - & - \\
\hline \multicolumn{9}{|l|}{$\begin{array}{l}\text { Epstein-Barr virus } \\
\text { DNA titer (copy/mL) }\end{array}$} \\
\hline$<10,000$ & Reference & & Reference & & Reference & & Reference & \\
\hline $10,000-99,999$ & $1.34(0.86-2.08)$ & 0.196 & $1.14(0.73-1.78)$ & 0.561 & $1.48(1.03-2.12)$ & 0.036 & $1.34(0.93-1.93)$ & 0.123 \\
\hline$\geq 100,000$ & $2.04(1.17-3.56)$ & 0.012 & $1.90(1.08-3.35)$ & 0.026 & $2.08(1.29-3.35)$ & 0.003 & $2.00(1.23-3.24)$ & 0.005 \\
\hline \multicolumn{9}{|l|}{$\begin{array}{l}\text { Neutrophil-to-lymphocyte } \\
\text { ratio }^{\text {a) }}\end{array}$} \\
\hline$\geq 2.35$ vs. $<2.35$ (ref.) & $1.20(0.80-1.79)$ & 0.380 & - & - & $1.42(1.01-1.98)$ & 0.041 & $1.40(1.00-1.96)$ & 0.050 \\
\hline \multicolumn{9}{|c|}{ Clinical stage (8th edition) $)^{b}$} \\
\hline IVA vs. III (ref.) & $3.12(1.95-4.99)$ & $<0.001$ & $2.95(1.83-4.74)$ & $<0.001$ & $2.23(1.55-3.21)$ & $<0.001$ & $2.04(1.41-2.95)$ & $<0.001$ \\
\hline \multicolumn{9}{|l|}{ Chemotherapy regimen } \\
\hline $\mathrm{PF}$ & Reference & & - & & Reference & & - & \\
\hline $\mathrm{TPF}$ & $0.82(0.50-1.35)$ & 0.436 & - & - & $0.85(0.56-1.28)$ & 0.427 & - & - \\
\hline $\mathrm{TP}$ & $0.76(0.45-1.28)$ & 0.302 & - & - & $0.73(0.47-1.14)$ & 0.166 & - & - \\
\hline \multicolumn{9}{|l|}{ IC-1-induced neutropenia } \\
\hline Absent & Reference & & Reference & & Reference & & Reference & \\
\hline Grade 1-2 & $1.84(1.15-2.96)$ & 0.012 & $1.86(1.15-2.98)$ & 0.011 & $1.63(1.11-2.39)$ & 0.012 & $1.63(1.11-2.39)$ & 0.012 \\
\hline Grade 3-4 & $2.25(1.35-3.76)$ & 0.002 & $2.29(1.38-3.83)$ & 0.001 & $1.66(1.07-2.55)$ & 0.023 & $1.72(1.11-2.66)$ & 0.015 \\
\hline Grade 3-4 vs. 1-2 (ref.) & $1.22(0.75-1.99)$ & 0.420 & $1.23(0.76-2.00)$ & 0.395 & $1.02(0.66-1.56)$ & 0.945 & $1.05(0.68-1.61)$ & 0.810 \\
\hline
\end{tabular}

$\mathrm{HR}$, hazard ratio; CI, confidence interval; AHR, adjusted hazard ratio; ref., reference; WHO, World Health Organization; PF, cisplatin-5-fluorouracil; TPF, docetaxel-cisplatin-5-fluorouracil; TP, docetaxel-cisplatin; IC-1, the first-cycle of induction chemotherapy. ${ }^{a}$ All variables were measured before treatment, b) 8 th edition of the Union for International Cancer Control / American Joint Committee on Cancer staging system. 


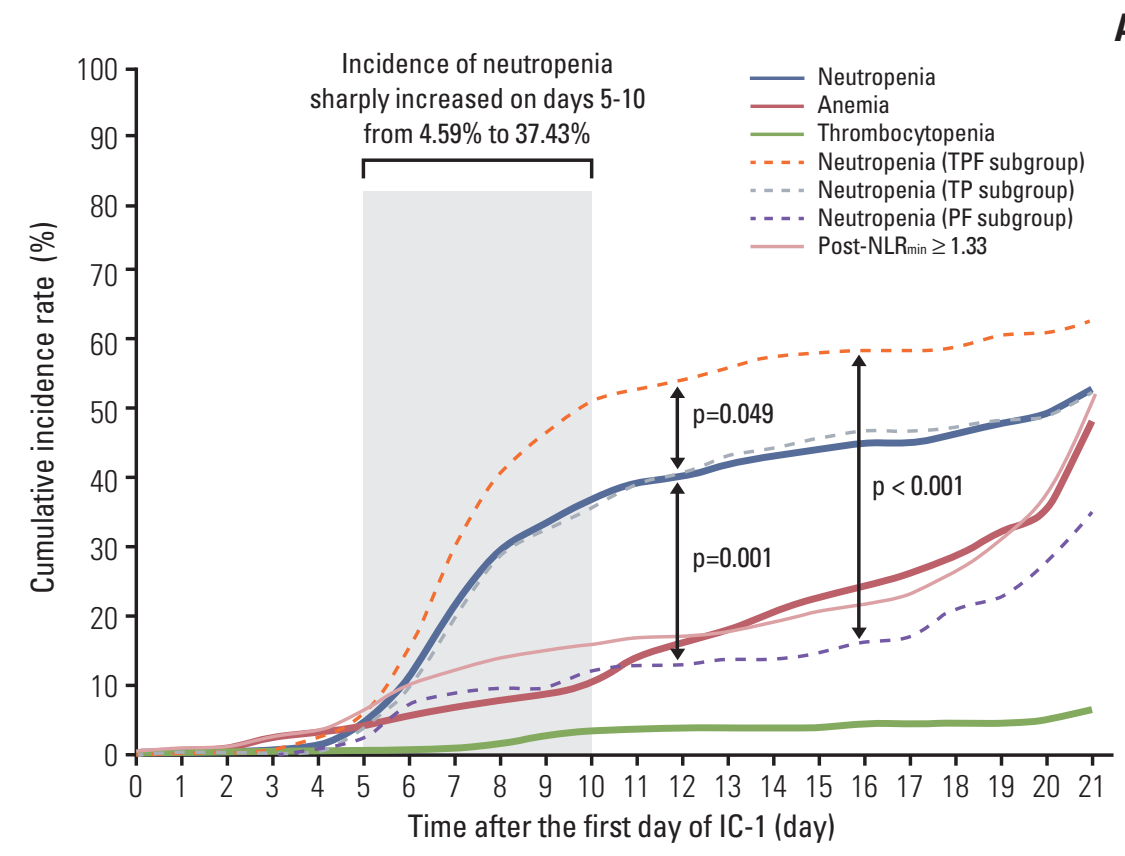

A

B

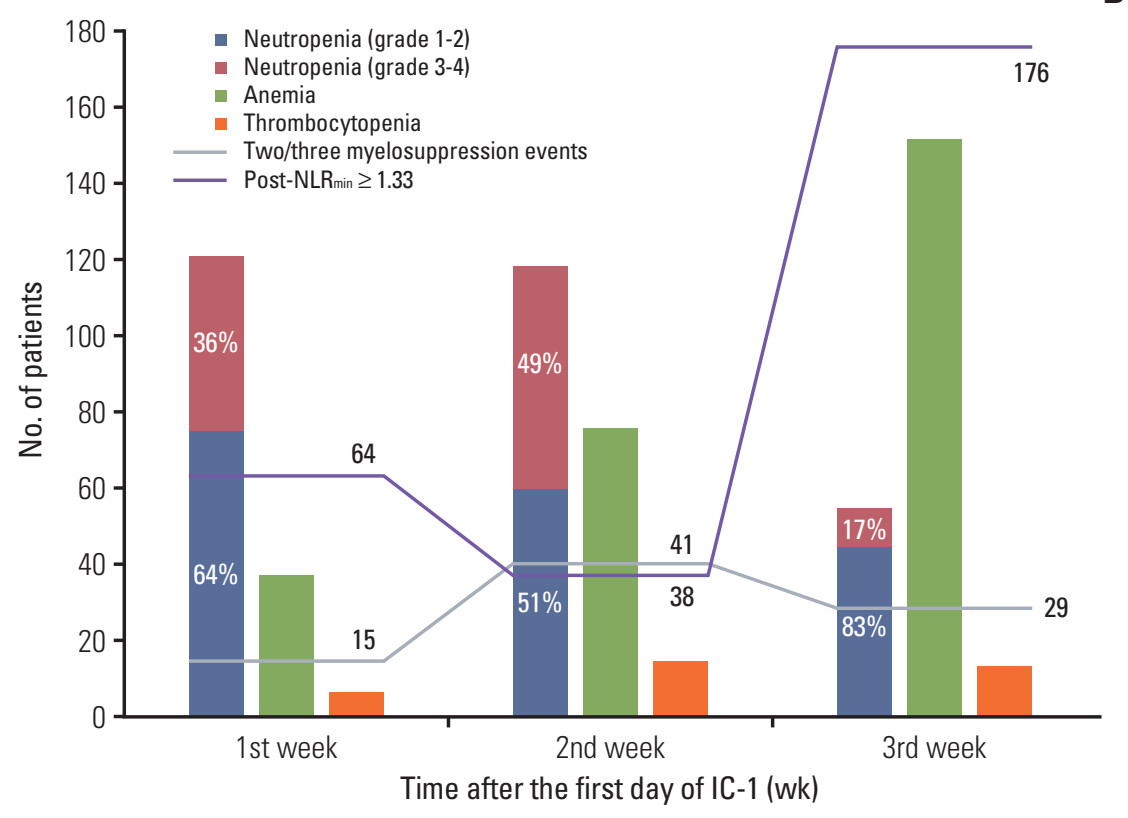

Fig. 2. Cumulative incidence rate (A) and frequency distribution (B) of myelosuppression events and minimal value of the post-treatment neutrophil-to-lymphocyte ratio (post-NLR $R_{\min }$ ). (A) Kaplan-Meier estimates of the time-to-occurrence over the 21-day cycle of the first cycle of induction chemotherapy (IC-1). (B) Histogram and line chart of number of patients suffering myelosuppression events and high post-NLR $\min$ in first/ second/third weeks of IC-1. TPF, docetaxel-cisplatin-5-fluorouracil; TP, docetaxel-cisplatin; PF, cisplatin-5-fluorouracil. 
Table 3. Univariate analysis of IC-1-induced neutropenia, timing of neutropenia, number of myelosuppression events, and post-NLR $R_{\min }$

\begin{tabular}{|c|c|c|c|c|c|c|c|c|c|}
\hline \multirow[b]{2}{*}{ Variable } & \multirow[b]{2}{*}{ No. $(\%)$} & \multicolumn{4}{|c|}{ Overall survival } & \multicolumn{4}{|c|}{ Disease-free survival } \\
\hline & & $\begin{array}{c}\text { 3-Year } \\
(\%)\end{array}$ & $\begin{array}{c}5 \text {-Year } \\
(\%)\end{array}$ & $\begin{array}{c}\text { HR } \\
(95 \% \mathrm{CI})\end{array}$ & p-value & $\begin{array}{c}\text { 3-Year } \\
(\%)\end{array}$ & $\begin{array}{c}\text { 5-Year } \\
(\%)\end{array}$ & $\begin{array}{c}\text { HR } \\
(95 \% \mathrm{CI})\end{array}$ & p-value \\
\hline \multicolumn{10}{|l|}{ IC-1-induced neutropenia } \\
\hline Absent & $253(46.4)$ & 95.6 & 88.0 & Reference & & 84.4 & 79.7 & Reference & \\
\hline Occurred & $292(53.6)$ & 87.2 & 78.2 & $2.00(1.30-3.06)$ & 0.001 & 75.5 & 69.8 & $1.64(1.16-2.31)$ & 0.005 \\
\hline \multicolumn{10}{|l|}{ Timing of neutropenia ${ }^{\text {a) }}$} \\
\hline Absent & $253(46.4)$ & 95.6 & 88.0 & Reference & & 84.4 & 79.7 & Reference & \\
\hline 1st week & $120(22.0)$ & 88.2 & 77.9 & $2.10(1.27-3.49)$ & 0.004 & 75.6 & 68.9 & $1.68(1.11-2.56)$ & 0.015 \\
\hline 2nd week & $118(21.7)$ & 88.8 & 79.9 & $1.80(1.06-3.07)$ & 0.030 & 76.9 & 75.1 & $1.35(0.86-2.13)$ & 0.187 \\
\hline 3rd week & $54(9.9)$ & 81.2 & 75.5 & $2.20(1.15-4.20)$ & 0.017 & 71.9 & 60.5 & $2.20(1.32-3.66)$ & 0.002 \\
\hline 2nd vs. 1st week (ref.) & - & - & - & $0.85(0.50-1.67)$ & 0.566 & - & - & $0.81(0.50-1.30)$ & 0.375 \\
\hline 3rd vs. 1st week (ref.) & - & - & - & $1.05(0.54-2.01)$ & 0.894 & - & - & $1.30(0.77-2.23)$ & 0.323 \\
\hline 3rd vs. 2nd week (ref.) & - & - & - & $1.22(0.62-2.40)$ & 0.564 & - & - & $1.61(0.93-2.86)$ & 0.088 \\
\hline \multicolumn{10}{|l|}{ Myelosuppression events ${ }^{\text {b) }}$} \\
\hline Group 1 (none) & $150(27.5)$ & 95.2 & 86.6 & Reference & & 83.1 & 78.1 & Reference & \\
\hline Group 2 (one) & $215(39.4)$ & 92.5 & 83.6 & $1.29(0.75-2.23)$ & 0.362 & 81.2 & 76.1 & $1.08(0.96-1.67)$ & 0.747 \\
\hline Group 3 (two/three) & $180(33.0)$ & 86.0 & 78.6 & $1.81(1.06-3.09)$ & 0.029 & 74.9 & 69.2 & $1.53(1.00-2.35)$ & 0.050 \\
\hline \multicolumn{10}{|l|}{ Post-NLR $_{\min }$} \\
\hline$<1.33$ & $267(49.0)$ & 93.9 & 86.3 & Reference & & 82.6 & 79.0 & Reference & \\
\hline$\geq 1.33$ & $278(51.0)$ & 88.4 & 79.4 & $1.42(0.95-2.13)$ & 0.087 & 76.8 & 69.9 & $1.51(1.08-2.12)$ & 0.016 \\
\hline
\end{tabular}

IC-1, the first cycle of induction chemotherapy; post-NLR ${ }_{\min }$, minimal value of the post-treatment neutrophil-to-lymphocyte ratio; HR, hazard ratio; CI, confidence interval; ref., reference. a) First day of IC-1 until most severe neutropenia, b)Three myelosuppression events, i.e., neutropenia, anemia, and thrombocytopenia, were analyzed in this study.

significantly different between grade 1-2 and grade 3-4 neutropenia $(\mathrm{p}=0.420$ and $\mathrm{p}=0.952)$. Similar results were observed in the subgroup receiving PF. Patients receiving TPF had equivalent OS/DFS, regardless of no/grade 1-2/ grade 3-4 neutropenia (all $p>0.05$ ). For patients receiving $\mathrm{TP}$, no neutropenia was associated with better OS/DFS vs. grade $3-4$ neutropenia $(\mathrm{p}=0.026$ and $\mathrm{p}=0.037$ ) but not $\mathrm{vs}$. grade 1-2 neutropenia.

In univariate analysis, WHO histologic type I-II, EBV DNA titer $\geq 100,000$ copies $/ \mathrm{mL}$, clinical stage IVA, and grade 1-2/grade 3-4 neutropenia were associated with poor OS/DFS; NLR $\geq 2.35$ was also associated with poor DFS (all $\mathrm{p}<0.05$ ) (Table 2). All aforementioned variables retained independent significance after adjustment in multivariate analysis (all $\mathrm{p}<0.05$ ) (Table 2). Notably, OS/DFS were not significantly different between patients with grade 1-2 vs. 3-4 neutropenia $(\mathrm{p}=0.395$ and $\mathrm{p}=0.810)$.

\section{Occurrence of myelosuppression and post-NLR $R_{\min }$ dur- ing IC-1}

The cumulative incidence of neutropenia, anemia, throm- bocytopenia, and high post-NLR $R_{\min }(\geq 1.33)$ was $53.58 \%$, $48.81 \%, 6.61 \%$, and $51.01 \%$ on the 21 st day of IC-1. The incidence of neutropenia sharply increased on days 5-10, then continued to gradually increase; the incidence of anemia and high post-NLR $R_{\min }$ gradually increased; and the incidence of thrombocytopenia remained low but gradually increased over the 21-day cycle of IC-1 (Fig. 2A). TPF regimen was more likely to lead to neutropenia compare with TP $(p=0.049)$ and PF $(p=0.001)$. The trend of cumulative incidence of myelosuppression events and high post-NLR $R_{\text {min }}$ were in accordance with the frequency distributions in Fig. 2B. Simultaneous multiple myelosuppression events were more likely to occur in the second week of IC-1, while high post$\mathrm{NLR}_{\text {min }}$ tended to occur in the third week of IC-1.

\section{Effect of IC-1-induced neutropenia, timing of neutrope- nia, number of myelosuppression events, and high post- NLR $_{\min }$ on survival}

As patients with grade 1-2 and 3-4 neutropenia had comparable survival outcomes, these groups were combined to a single neutropenia group. Univariate analysis (Table 3) 

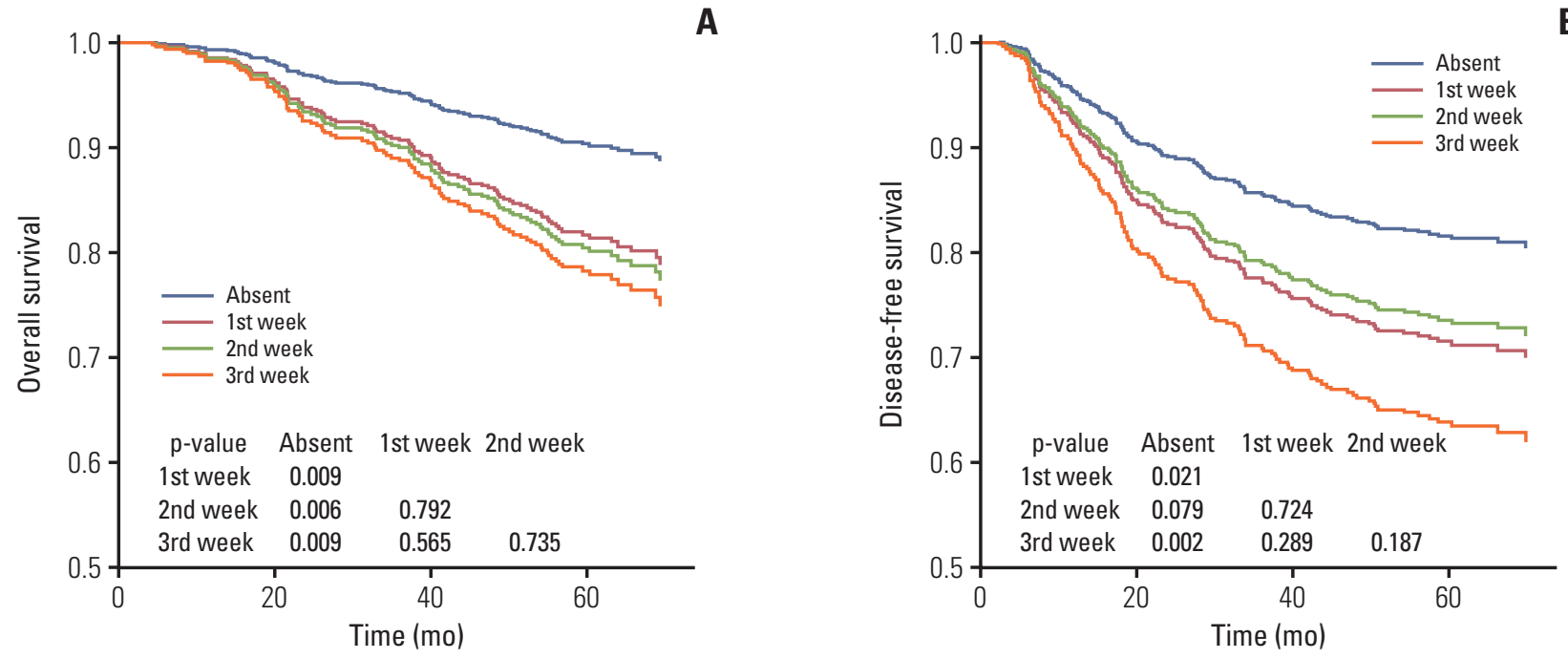

$\begin{array}{rrrrr}\text { No. at risk } & & & & \\ \text { Absent } & 252 & 244 & 201 & 84 \\ \text { 1st week } & 120 & 113 & 91 & 38 \\ \text { 2nd week } & 118 & 111 & 82 & 32 \\ \text { 3rd week } & 54 & 49 & 38 & 15\end{array}$

A

No. at risk

$\begin{array}{rrrrr}\text { Absent } & 252 & 221 & 175 & 76 \\ \text { 1st week } & 120 & 101 & 77 & 34 \\ \text { 2nd week } & 118 & 98 & 72 & 30 \\ \text { 3rd week } & 54 & 41 & 33 & 13\end{array}$

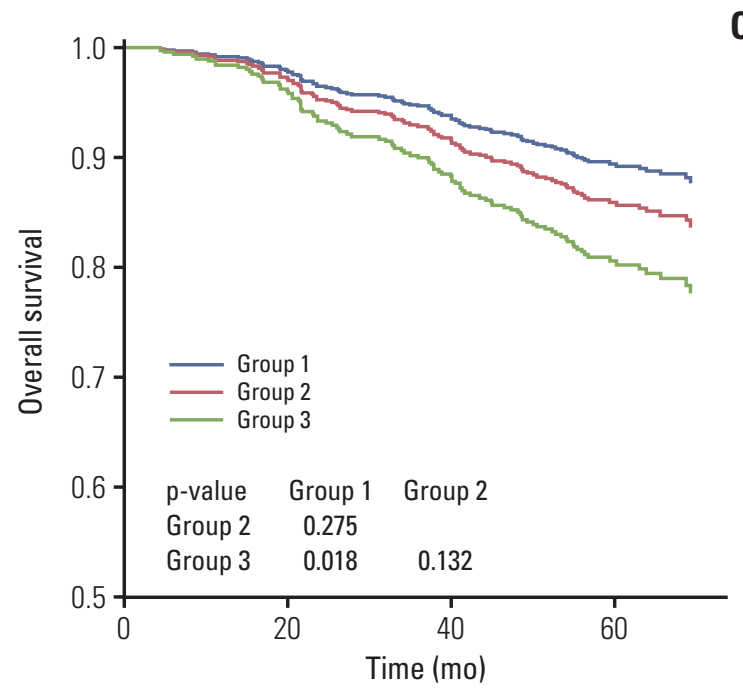

No. at risk

$\begin{array}{lllll}\text { Group 1 } & 149 & 143 & 118 & 50 \\ \text { Group 2 } & 214 & 205 & 160 & 61 \\ \text { Group 3 } & 180 & 168 & 133 & 59\end{array}$

C

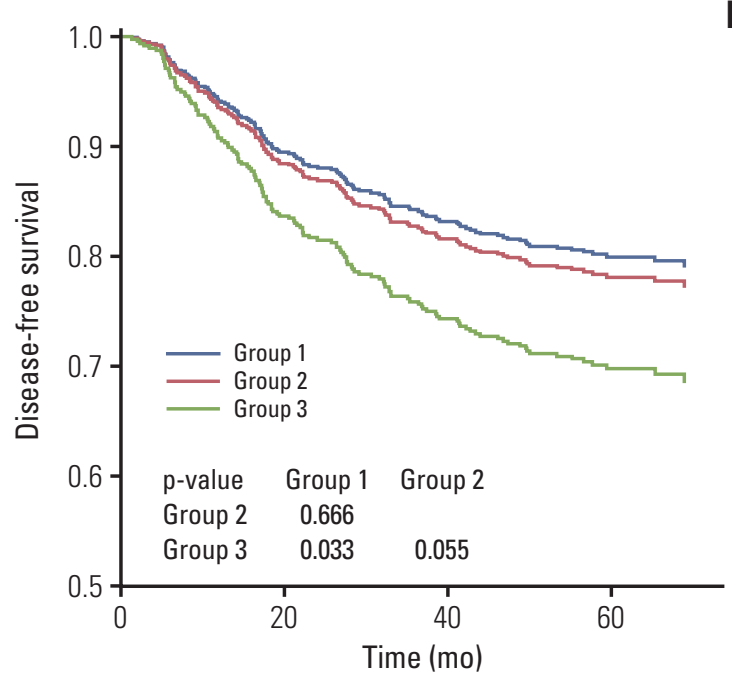

No. at risk

$\begin{array}{lllll}\text { Group 1 } & 149 & 128 & 103 & 46 \\ \text { Group 2 } & 214 & 186 & 139 & 56 \\ \text { Group 3 } & 180 & 147 & 115 & 51\end{array}$

Fig. 3. Kaplan-Meier survival curves for overall survival (A, C, E) and disease-free survival (B, D, F) after adjustment for covariates. Patients were stratified by timing of neutropenia (absent and first/ second/third week of the first cycle of induction chemotherapy) (A, B), number of myelosuppression events (group 1, none; group 2, one; group 3, two / three) (C, D), and minimal value of the post-treatment neutrophil-to-lymphocyte ratio (post-NLR $\min _{1}<1.33$ and $\geq 1.33$ ) (E, F). p-values for overall survival (disease-free survival) were calculated using multivariate Cox regression analyses adjusted for World Health Organization histologic type, Epstein-Barr virus DNA titer and clinical stage (all of these covariates plus neutrophil-to-lymphocyte ratio). (Continued to the next page) 


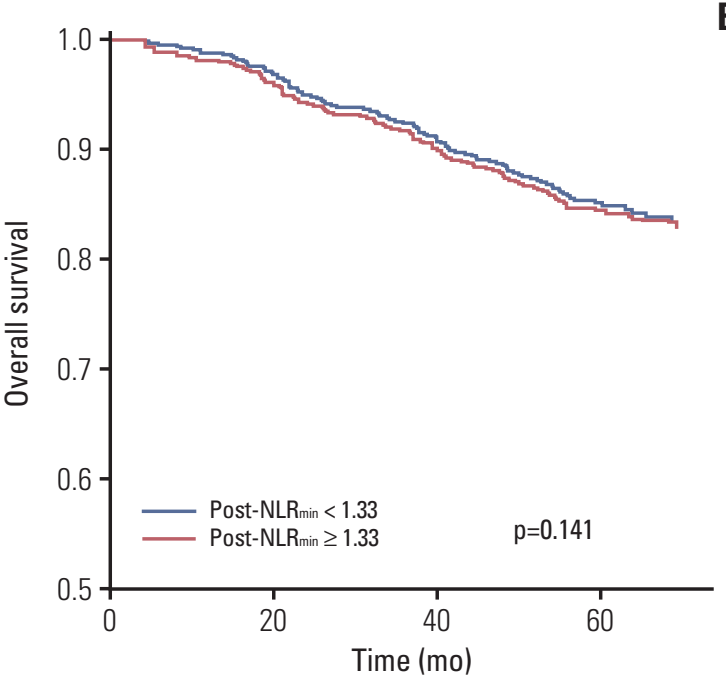

$\mathbf{E}$

No. at risk

$\begin{array}{lllll}<1.33 & 266 & 257 & 205 & 80 \\ \geq 1.33 & 277 & 260 & 205 & 79\end{array}$

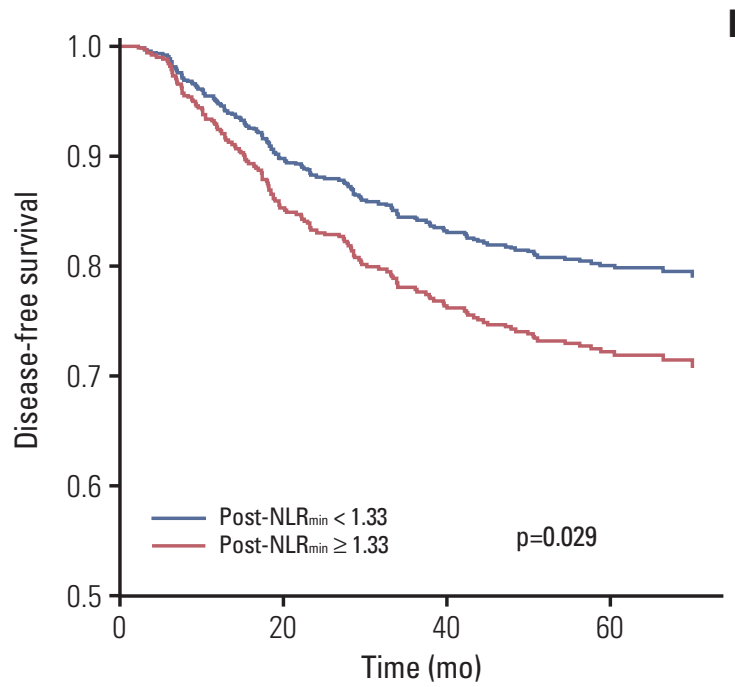

$\begin{array}{rrrrr}\text { No. at risk } & & & & \\ <1.33 & 266 & 232 & 180 & 70 \\ \geq 1.33 & 277 & 229 & 177 & 72\end{array}$

Fig. 3. (Continued from the previous page)

\begin{tabular}{|c|c|c|c|c|c|c|}
\hline Subgroup & $\begin{array}{c}\text { No. of } \\
\text { patients }\end{array}$ & $\begin{array}{c}\text { Occurred } \\
(\%)\end{array}$ & $\begin{array}{l}\text { Absent } \\
(\%, \text { ref. })\end{array}$ & & $\operatorname{AHR}(95 \% \mathrm{CI})$ & $\mathrm{p}$-value \\
\hline Overall survival & & & & 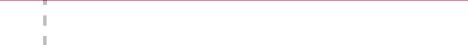 & & \\
\hline PF regimen & 121 & $43(35.5)$ & $78(64.5)$ & - & $3.53(1.03-12.14)$ & 0.046 \\
\hline TPF regimen & 230 & $146(63.5)$ & $84(36.5)$ & $\mapsto$ & $1.04(0.52-2.10)$ & 0.906 \\
\hline TP regimen & 194 & $103(53.1)$ & 91 (46.9) & —— & $3.13(0.75-13.12)$ & 0.119 \\
\hline Overall & 545 & $292(53.6)$ & $253(46.4)$ & $\longmapsto$ & $2.44(1.55-3.82)$ & $<0.001$ \\
\hline \multicolumn{4}{|c|}{ Disease-free survival } & i & & \\
\hline PF regimen & 121 & $43(35.5)$ & $78(64.5)$ & ए尸 & $3.90(1.74-8.75)$ & 0.001 \\
\hline TPF regimen & 230 & $146(63.5)$ & $84(36.5)$ & $\mapsto$ & $0.87(0.46-1.65)$ & 0.671 \\
\hline TP regimen & 194 & $103(53.1)$ & $91(46.9)$ & $\longmapsto$ & $5.09(1.68-15.44)$ & 0.004 \\
\hline Overall & 545 & $292(53.6)$ & $253(46.4)$ & :— & $2.05(1.42-2.97)$ & $<0.001$ \\
\hline
\end{tabular}

Favors survival $\longleftarrow \longrightarrow$ Favors death

Fig. 4. Forest plots depicting adjusted hazard ratios (AHRs) (squares) and 95\% confidence intervals (CIs) (bars) of the association between the first-cycle of induction chemotherapy (IC-1)-induced neutropenia and overall/disease-free survival. Blue and red squares individually indicate all patients and subgroups receiving different regimens. AHRs for overall survival (disease-free survival) was adjusted for timing of neutropenia, IC-1-induced anemia, IC-1-induced thrombocytopenia, minimal value of the post-treatment neutrophil-to-lymphocyte ratio, World Health Organization histologic type, Epstein-Barr virus DNA titer and clinical stage (all covariates plus neutrophil-to-lymphocyte). Ref., reference; PF, cisplatin-5-fluorouracil; TPF, docetaxel-cisplatin-5-fluorouracil; TP, docetaxel-cisplatin. 
revealed neutropenia had a negative effect on OS/DFS vs. no neutropenia $(\mathrm{p}=0.001$ and $\mathrm{p}=0.005)$. Patients with neutropenia had almost $10 \%$ lower 3/5-year OS/DFS than patients without neutropenia. Significantly poorer OS/DFS were observed for patients with neutropenia during the first, second, and third weeks of IC- 1 vs. no neutropenia (all $p<0.05$ ), with the exception of "second week" vs. "absent" for DFS; "second week" was associated with higher 3/5-year OS / DFS than "first week" / "third week," though the differences were non-significant (all $p>0.05$ ). Group 3 had significantly poorer OS / DFS than group $1(\mathrm{p}=0.029$ and $\mathrm{p}=0.050)$; no significant differences in OS / DFS existed between group 1 and group $2(p=0.362$ and $p=0.747)$. Patients with post$\mathrm{NLR}_{\min } \geq 1.33$ had poorer DFS ( $\mathrm{p}=0.016$ ) and equivalent $\mathrm{OS}$ ( $\mathrm{p}=0.087$ ) compared to patients with post-NLR $\mathrm{Rin}_{\min }<1.33$.

In multivariate analysis, AHRs for OS (DFS) were adjusted for WHO histologic type, EBV DNA titer and clinical stage (all of these covariates plus NLR) (Fig. 3A-F). The threeweeks classification failed to separate OS/DFS (all p >0.05). Two/three myelosuppression events was validated as negative prognostic factor for OS / DFS ( $\mathrm{p}=0.018$ and $\mathrm{p}=0.033$ ); high post-NLR $\min (\geq 1.33)$ was only significant in predicting poor DFS $(\mathrm{p}=0.029)$. We included timing of neutropenia (absent/first/ second/third week), IC-1-induced anemia and thrombocytopenia (absent/occurred), and post-NLR $R_{\min }$ $(\geq 1.33 /<1.33)$ as covariates in additional multivariate analysis. Neutropenia had a negative effect on OS (AHR, 2.44; $\mathrm{p}<0.001$ ) and DFS (AHR, 2.05; $\mathrm{p}<0.001$ ) (Fig. 4). Similar effects were observed in patients receiving $\mathrm{PF}$ (OS, $\mathrm{p}=0.046$; DFS, $\mathrm{p}=0.001$ ) or TP (DFS, $\mathrm{p}=0.004)$, but not TPF (OS, $\mathrm{p}=0.906$; DFS, $\mathrm{p}=0.671$ ) (Fig. 4).

\section{Discussion}

This first attempt to explore the prognostic value of the occurrence and severity of IC-1-induced neutropenia in LANPC validated two/three myelosuppression events and high post-NLR $R_{\min }$ have significant negative effects on OS and/or DFS; the timing of neutropenia had no significant effect. This information could improve prognostication and selection of high-risk patients, and indicate some possible clinical implications. Routine blood test (RBT) is widely used in primary, secondary, and even tertiary hospitals; the diagnostic criteria for each item of RBT are basically identical among different hospitals. Moreover, RBT is suitable for almost all patients, because of its low price, simplicity, and safety. Thus, dynamic monitoring of ANC, lymphocyte, $\mathrm{Hb}$, and PLT over the whole course of treatment is convenient and has clinical applicability. Patients who experience neu- tropenia or improved post-NLR $\mathrm{min}_{\min }$ during IC-1 can be screened out as early as possible. After comprehensive evaluation, those high-risk patients can receive timely supportive treatment, targeted interventions (e.g., prophylactic G-CSF in future cycles), and modification of treatment (e.g., reducing chemotherapy dose), with the aim of improving patients' survival benefits.

Several pretreatment inflammatory-immune biomarkers can predict prognosis, such as NLR, platelet-to-lymphocyte ratio, and lymphocyte-to-monocyte ratio [22-24]. Of which, pretreatment NLR can predict survival outcomes and has been used to generate predictive nomograms in NPC $[17,25]$. Elevated pretreatment NLR indicates a disordered immune response, which is associated cancer development and progression [18]. However, the relationship between post-treatment inflammatory-immune biomarkers (e.g., CIN and post-NLR) and survival remains crude. Although previous studies explored the prognostic value of CIN in other cancers, few focused on IC-1-induced neutropenia [7-13]. Moreover, the design of these studies limits their reliability and applicability; for example, strict inclusion/exclusion criteria (e.g., age $\geq 70$ years [8]), landmark/ subset analysis (i.e., only subgroups analyzed $[7,9,10])$ and missing pretreatment NLR data. These factors may also explain the positive $[7,8]$, mixed [9], non-significant $[10,11]$, and negative results $[12,13]$ of the aforementioned studies. Therefore, this real-world study including data on comprehensive pretreatment biomarkers (e.g., NLR, EBV DNA titer, VCA-IgA, and EA-IgA) provides a robust assessment of the associations between IC-1induced neutropenia and survival in LANPC.

IC-1-induced neutropenia, but not the timing of neutropenia, had prognostic value for poor survival in patients with LANPC receiving TP/PF. In contrast, previous studies suggested CIN after multiple chemotherapy cycles $[7,8]$ and early-onset $[5,6]$ were associated with a better prognosis. There are three possible explanations for these discrepancies. First, CIN after multiple cycles was regarded as an indicator of high drug activity and excellent treatment efficiency [7]. Patients with CIN were considered to have revived more intensive treatment and therefore achieve better survival outcomes. However, a higher dose of chemotherapy does not always improve prognosis. Peng et al. [26] reported a high cumulative cisplatin dose $\left(\geq 240 \mathrm{mg} / \mathrm{m}^{2}\right)$ did not improve OS/DFS (vs. low cumulative dose) in LANPC. Moreover, it is inappropriate to evaluate the cumulative dose and intensity after only the first cycle of IC. Hence, IC-1-induced neutropenia has limited ability to indicate treatment efficacy. Second, chemotherapy-related hematological toxicities, such as myelosuppression events and lymphopenia, can indicate deterioration of host immune function. Past evidences have reported that immunosuppressive agents increase cancer risk in patients receiving renal transplants, while additional 
immunestimulants improve survival in advanced Ewing sarcoma [16,27]. Although neutropenia can easily lead to low post-NLR $R_{\min }$, we cannot simply assume that the negative effect of neutropenia is contradictory to the positive effect of low post-NLR ${ }_{\min }$, since post-NLR $\mathrm{min}_{\text {is }}$ a joint biomarker and determined by neutrophil and lymphocyte counts. In our study, only $72.7 \%$ of patients with low post-NLR $\mathrm{Nin}_{\min }$ experienced IC-1-induced neutropenia; the remaining patients had normal ANC and elevated lymphocyte counts. Among patients with NPC treated by CCRT alone, treatment-related lymphopenia was associated with poorer survival and higher risk of death, disease progression, and distant metastasis [28]. Thus, patients with IC-1-induced neutropenia and / or high post-NLR min $_{\text {are }}$ predisposed to vulnerable host immune function, indicating poorer survival. Third, we only analyzed myelosuppression events during the 21-day cycle of IC-1; the aforementioned studies with positive results undertook longer observations over more than two cycles.

Interestingly, IC-1-induced neutropenia was not associated with survival among patients receiving TPF. The TPF regimen, a potent IC based on three chemotherapeutic agents, has demonstrated promising results in LANPC [2]. TPF takes longer to administer than TP, since 5-fluorouracil is given via continuous intravenous infusion on days 1-5, while docetaxel and cisplatin are administered on day 1 . Moreover, our study showed that TPF IC-1 can lead to significantly higher incidence of neutropenia than $\mathrm{PF} / \mathrm{TP}$ regimen (Fig. 2A). Therefore, in clinical practice, patients receiving TPF usually receive from more meticulous nursing care, longer observation, and additional supportive treatments. Such intensive management may help to prevent further deterioration and promote recovery. Meanwhile, the relationship between TPF IC-1-induced neutropenia and survival was influenced, to some extent, by those medical interventions.

This study has several limitations. First, although we included pretreatment NLR and post-NLR $R_{\min }$, the optimal cutoff has not been identified. For pretreatment NLR, different research centers use varied levels, including 2.70 [29], 3.00 [24], and 3.73 [30]. Moreover, not all cutoff values were proven significant [24]. Although we used and validated the median of pretreatment NLR and post-NLR ${ }_{\text {min }}$ as the cutoff for DFS, these values failed to significantly discriminate different OS. Similarly, standardized procedures and assay harmonization for pretreatment EBV DNA are not yet established. Thus, cutoff selection may result in bias and error. Second, the sample sizes for certain subgroups were relatively small, especially patients receiving PF (neutropenia, $\mathrm{n}=43$; absent, $\mathrm{n}=78$ ) and with neutropenia in the third week $(n=54)$. Future large-scale prospective studies are required to further address this issue. Last but not least, although RBTs were required once a week, the precise timings varied from person to person, which may modify the distribution of myelosuppression.

\section{Electronic Supplementary Material}

Supplementary materials are available at Cancer Research and Treatment website (http://www.e-crt.org).

\section{Conflicts of Interest}

Conflict of interest relevant to this article was not reported.

\section{Acknowledgments}

This work was supported by grants from the National Science \& Technology Pillar Program during the Twelfth Five-year Plan Period (No. 2014BAI09B10), the Science and Technology Project of Guangzhou City, China (No. 14570006), the Planned Science and Technology Project of Guangdong Province, China (No. 2013B020400004), the Health \& Medical Collaborative Innovation Project of Guangzhou City, China (No. 201400000001), and the National Natural Science Foundation of China (No. 81230056).

\section{References}

1. Mao YP, Xie FY, Liu LZ, Sun Y, Li L, Tang LL, et al. Re-evaluation of 6th edition of AJCC staging system for nasopharyngeal carcinoma and proposed improvement based on magnetic resonance imaging. Int J Radiat Oncol Biol Phys. 2009;73:1326-34.

2. Sun Y, Li WF, Chen NY, Zhang N, Hu GQ, Xie FY, et al. Induction chemotherapy plus concurrent chemoradiotherapy versus concurrent chemoradiotherapy alone in locoregionally advanced nasopharyngeal carcinoma: a phase 3 , multicentre, randomised controlled trial. Lancet Oncol. 2016;17:1509-20.

3. Kuderer NM, Dale DC, Crawford J, Cosler LE, Lyman GH. Mortality, morbidity, and cost associated with febrile neutropenia in adult cancer patients. Cancer. 2006;106:2258-66.

4. Lyman GH, Kuderer NM, Crawford J, Wolff DA, Culakova E, Poniewierski MS, et al. Predicting individual risk of neutropenic complications in patients receiving cancer chemotherapy. Cancer. 2011;117:1917-27.

5. Chen Y, Wang Y, Shi Y, Dai G. Timing of chemotherapy- 
induced neutropenia predicts prognosis in metastatic colon cancer patients: a retrospective study in mFOLFOX6 -treated patients. BMC Cancer. 2017;17:242.

6. Lee CY, Park SY, Shin TR, Park YB, Kim CH, Jang SH, et al. Early-onset neutropenia during perioperative chemotherapy is predictive of increased survival in patients with completely resected non-small cell lung cancer: a retrospective analysis. Anticancer Res. 2013;33:2755-61.

7. Kishida Y, Kawahara M, Teramukai S, Kubota K, Komuta K, Minato K, et al. Chemotherapy-induced neutropenia as a prognostic factor in advanced non-small-cell lung cancer: results from Japan Multinational Trial Organization LC00-03. Br J Cancer. 2009;101:1537-42.

8. Di Maio M, Gridelli C, Gallo C, Shepherd F, Piantedosi FV, Cigolari $S$, et al. Chemotherapy-induced neutropenia and treatment efficacy in advanced non-small-cell lung cancer: a pooled analysis of three randomised trials. Lancet Oncol. 2005;6:669-77.

9. Tewari KS, Java JJ, Gatcliffe TA, Bookman MA, Monk BJ. Chemotherapy-induced neutropenia as a biomarker of survival in advanced ovarian carcinoma: an exploratory study of the Gynecologic Oncology Group. Gynecol Oncol. 2014;133: 439-45.

10. Kim JJ, Park JY, Kim DY, Kim JH, Kim YM, Nam JH, et al. Is chemotherapy-induced neutropenia a prognostic factor in patients with ovarian cancer? Acta Obstet Gynecol Scand. 2010;89:623-8.

11. Kumpulainen EJ, Hirvikoski PP, Johansson RT. Neutropenia during adjuvant chemotherapy of breast cancer is not a predictor of outcome. Acta Oncol. 2009;48:1204-6.

12. Bogani G, Sabatucci I, Maltese G, Lecce F, Signorelli M, Martinelli $\mathrm{F}$, et al. Chemotherapy-related leukopenia as a biomarker predicting survival outcomes in locally advanced cervical cancer. Eur J Obstet Gynecol Reprod Biol. 2017;208: 41-5.

13. Miyoshi N, Yano M, Takachi K, Kishi K, Noura S, Eguchi H, et al. Myelotoxicity of preoperative chemoradiotherapy is a significant determinant of poor prognosis in patients with T4 esophageal cancer. J Surg Oncol. 2009;99:302-6.

14. Blay JY, Chauvin F, Le Cesne A, Anglaret B, Bouhour D, Lasset C, et al. Early lymphopenia after cytotoxic chemotherapy as a risk factor for febrile neutropenia. J Clin Oncol. 1996;14: 636-43.

15. Crawford J, Dale DC, Lyman GH. Chemotherapy-induced neutropenia: risks, consequences, and new directions for its management. Cancer. 2004;100:228-37.

16. Wong G, Chapman JR. Cancers after renal transplantation. Transplant Rev (Orlando). 2008;22:141-9.

17. Xu C, Chen YP, Liu X, Li WF, Chen L, Mao YP, et al. Establishing and applying nomograms based on the 8th edition of the UICC/AJCC staging system to select patients with nasopharyngeal carcinoma who benefit from induction chemotherapy plus concurrent chemoradiotherapy. Oral Oncol. 2017;69:99-107.
18. Diakos CI, Charles KA, McMillan DC, Clarke SJ. Cancerrelated inflammation and treatment effectiveness. Lancet Oncol. 2014;15:e493-503.

19. Sherman RE, Anderson SA, Dal Pan GJ, Gray GW, Gross T, Hunter NL, et al. Real-world evidence: what is it and what can it tell us? N Engl J Med. 2016;375:2293-7.

20. Kaplan EL, Meier P. Nonparametric estimation from incomplete observations. J Am Stat Assoc. 1958;53:457-81.

21. Neyeloff JL, Fuchs SC, Moreira LB. Meta-analyses and Forest plots using a microsoft excel spreadsheet: step-by-step guide focusing on descriptive data analysis. BMC Res Notes. 2012;5:52.

22. Li XH, Chang H, Xu BQ, Tao YL, Gao J, Chen C, et al. An inflammatory biomarker-based nomogram to predict prognosis of patients with nasopharyngeal carcinoma: an analysis of a prospective study. Cancer Med. 2017;6:310-9.

23. Azab B, Mohammad F, Shah N, Vonfrolio S, Lu W, Kedia S, et al. The value of the pretreatment neutrophil lymphocyte ratio vs. platelet lymphocyte ratio in predicting the long-term survival in colorectal cancer. Cancer Biomark. 2014;14:303-12.

24. Ferrucci PF, Ascierto PA, Pigozzo J, Del Vecchio M, Maio M, Antonini Cappellini GC, et al. Baseline neutrophils and derived neutrophil-to-lymphocyte ratio: prognostic relevance in metastatic melanoma patients receiving ipilimumab. Ann Oncol. 2016;27:732-8.

25. Liang W, Shen G, Zhang Y, Chen G, Wu X, Li Y, et al. Development and validation of a nomogram for predicting the survival of patients with non-metastatic nasopharyngeal carcinoma after curative treatment. Chin J Cancer. 2016;35:98.

26. Peng H, Chen L, Zhang Y, Li WF, Mao YP, Zhang F, et al. Prognostic value of the cumulative cisplatin dose during concurrent chemoradiotherapy in locoregionally advanced nasopharyngeal carcinoma: a secondary analysis of a prospective phase III clinical trial. Oncologist. 2016;21:1369-76.

27. Burdach S, van Kaick B, Laws HJ, Ahrens S, Haase R, Korholz $\mathrm{D}$, et al. Allogeneic and autologous stem-cell transplantation in advanced Ewing tumors: an update after long-term followup from two centers of the European Intergroup study EICESS. Stem-Cell Transplant Programs at Dusseldorf University Medical Center, Germany and St. Anna Kinderspital, Vienna, Austria. Ann Oncol. 2000;11:1451-62.

28. Liu LT, Chen QY, Tang LQ, Guo SS, Guo L, Mo HY, et al. The prognostic value of treatment-related lymphopenia in nasopharyngeal carcinoma patients. Cancer Res Treat. 2018;50: 19-29.

29. Sun W, Zhang L, Luo M, Hu G, Mei Q, Liu D, et al. Pretreatment hematologic markers as prognostic factors in patients with nasopharyngeal carcinoma: neutrophil-lymphocyte ratio and platelet-lymphocyte ratio. Head Neck. 2016;38 Suppl 1:E1332-40.

30. An X, Ding PR, Wang FH, Jiang WQ, Li YH. Elevated neutrophil to lymphocyte ratio predicts poor prognosis in nasopharyngeal carcinoma. Tumour Biol. 2011;32:317-24. 